\title{
Dispersion of nano-silica in monomer casting nylon6 and its effect on the structure and properties of composites
}

\author{
L. F. Cai ${ }^{*}$, Z. Y. Lin $^{2}, H$. Qian $^{2}$ \\ ${ }^{1}$ Department of Environment and Life science, Putian University, No. 1133, Mid-Xueyuan St., Putian, Fujian Province \\ 351100, P. R. China \\ ${ }^{2}$ College of Materials Science and Engineering, Huaqiao University, No. 269, Chenghuabei Rd., Quanzhou, Fujian \\ Province 362021, P. R. China
}

Received 26 February 2010; accepted in revised form 24 April 2010

\begin{abstract}
To promote dispersion of nano-silica in monomer casting nylon6 (MC nylon6), nano-silica was dispersed in melted caprolactam with the assistance of ultrasound, anionic polymerization was then initiated to form silica/MC nylon6 in-situ nanocomposites. It was found that hydrogen bonds were formed between nano-silica and caprolactam, in the meantime, ultrasound helped to break the nanoparticles aggregations into smaller ones or even mono-dispersing particles. Therefore, the agglomerated nanoparticles were pulled apart and stabilized by caprolactam. Additionally, the rapid anionic polymerization of caprolactam also contributed to the avoidance of re-agglomeration and deposition of nanoparticles during the polymerization process, leading to the uniform distribution of nanoparticles in the polymer matrix. Mechanical tests indicated that the silica/MC nylon6 in-situ nanocomposites prepared according to the above strategy were simultaneously toughened, strengthened and stiffened. Thermogravimetric analysis (TGA) results showed that thermal stability of nanocomposites was notably improved compared to neat MC nylon6.
\end{abstract}

Keywords: nanocomposites, silica, MC nylon6, in-situ anionic polymerization

\section{Introduction}

Polymer-based nanocomposites have been reported as an exciting new class of materials for their markedly improved properties over neat polymers and micron-sized particle filled polymer composites $[1,2]$. However, the specific characteristics of the nanocomposites can only be effective provided that the nanoparticles are well dispersed in the matrix. Due to the strong tendency of nanoparticles to agglomerate, manufacturing composites with uniformly distributed nanoparticles becomes a challenging task [3].

To break down these nanoparticles aggregations and manufacture nano-structural composites, researchers mainly focus on the following three approaches: 1) sol-gel processing [4], 2) melt compounding [5-7], and 3) in-situ polymerization with the presence of nanoparticles [8,9]. During sol-gel process, the formation of a crosslinking network of organic metal oxides makes it difficult to process, and it is a disadvantage that limits the application of this method. Melt compounding is one of the most conventional processing methods. However, the previously synthesized polymers would inevitably undergo some degradation during processing. Comparatively, in-situ polymerization with the presence of nanoparticles seems to be rather facile. In this method, nanoparticles are dispersed in monomer first, and then the mixture is polymerized using a technology similar to bulk polymerization. It is 
obvious that the most important factor that affects the properties of composites is the dispersion of nanoparticles.

According to Tadros [10], the dispersion of ultrafine particles in medium can be divided into three procedures:

1. Wetting of particles into dispersion medium;

2. Breaking of aggregations into mono-dispersing particles or smaller aggregations;

3. Stabilization of mono-dispersing particles and smaller aggregations against re-agglomeration.

With the enlightenment of Tadros's work, the authors of the present paper plan to prepare silica/ monomer casting nylon6 (MC nylon6) in-situ nanocomposites via anionic polymerization according to the following procedure: nano-silica was directly dispersed into melted caprolactam with the assistance of ultrasound, and then anionic polymerization was initiated to form the resulting nanocomposites. Such strategy was designed according to the following: (i) it was reported that particles with polar surface can be easily wetted by polar liquid [11]. Accordingly, nano-silica with polar silanol groups on the surface can be easily wetted by polar melted caprolactam containing amide group, (ii) the preparation of the nylon6/silica nanocomposites using in-situ anionic polymerization was also recently reported by Rusu and Rusu [12]. It is found that nano-silica was simply dispersed in the melted caprolactam by ordinary stirrer. Accordingly, although some of the nano-silica was homogeneously dispersed, there were still some bigger aggregations (ca. $200 \mathrm{~nm}$ in diameter) within the nylon6 matrix. Considering that the tremendous energy offered by ultrasonic is likely to break the nanoparticles aggregations into smaller ones or even mono-dispersing particles [13], in the present work, ultrasound was employed to disperse nanosilica in melted caprolactam. Therefore, the agglomerated nanoparticles would be pulled apart and coated with caprolactam, leading to the improved stability of smaller aggregations and mono-dispersing nanoparticles against re-agglomeration, and (iii) in comparison with classical hydrolytic polymerization of caprolactam which takes about 12-24 hrs [14, 15], the rapid anionic polymerization of caprolactam (usually within $1 \mathrm{hr}[12,16]$ ) also contributed to the avoidance of re-agglomeration and deposition of nanoparticles during the polymerization process, leading to the uniform dis-

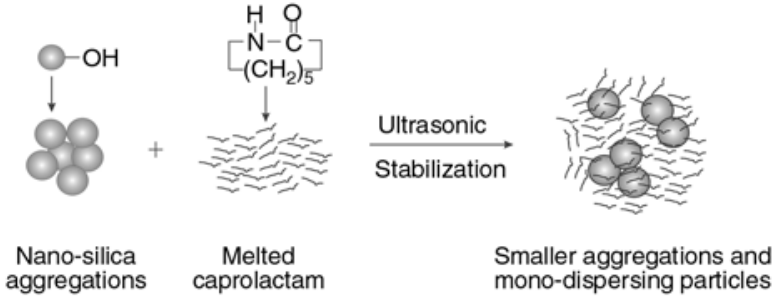

Anionic polymerization

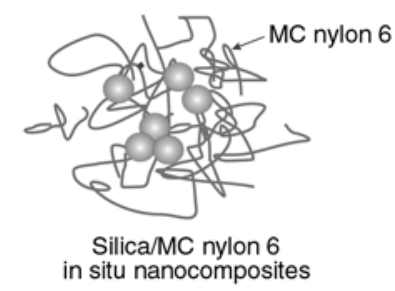

Figure 1. Schematic drawing of the proposed technology to prepare silica/MC nylon6 in-situ nanocomposites with uniformly distributed nanoparticles

tribution of nanoparticles in polymer matrix (Figure 1). Besides, it is well known that the anionic polymerization of caprolactam proceeds well below the melting temperature of the nylon6, which could avoid the degradation in comparison with conventional melt compounding. The present paper characterized the dispersion of nano-silica in the composite and preliminarily evaluated effect of nanosilica on the structure and properties of the MC nylon6 based nanocomposites.

\section{Experimental}

\subsection{Materials}

Nano-silica was supplied by Zhoushan Mingri Nanometer Material Corporation (Zhejiang, China) with an average diameter of $30 \pm 5 \mathrm{~nm}$. The particles were dried at $120^{\circ} \mathrm{C}$ under vacuum for $24 \mathrm{~h}$ to eliminate the physically absorbed and weakly chemically absorbed species.

$\varepsilon$-caprolactam (AR grade) was obtained from Baling Company, China Petroleum and Chemical Corporation (Hunan, China). Sodium hydroxide (AR grade) and 2,4-tolylene diisocyanate (2,4-TDI, CP grade, vacuum distillated before use) were obtained from Shanghai Chemical Reagents Corporation (Shanghai, China).

\subsection{Composites preparation}

Silica/MC nylon6 in-situ nanocomposites were obtained according to the following procedure: 
$100 \mathrm{~g}$ caprolactam was melted at $120^{\circ} \mathrm{C}$, then $0.40 \mathrm{~g}$ sodium hydroxide was added followed by vacuum treatment to eliminate the produced water, vacuum treatment was maintained about $30 \mathrm{~min}$ to confirm the thorough elimination of water. Then various contents of dried nano-silica (including 1.0, 2.0, 3.0 and $5.0 \mathrm{wt} \%$ ) was dispersed in this melt by the assistance of ultrasound (ultrasonic mixer with a power of $300 \mathrm{~W}$ and a frequency of $40 \mathrm{kHz}$ ) to obtain homogeneous mixture. Finally the mixture was heated to $150^{\circ} \mathrm{C}, 0.364 \mathrm{~g} \mathrm{2,4-TDI}$ was introduced as cocatalyst, and the anionic polymerization time was $60 \mathrm{~min}$.

\subsection{Characterization}

Fourier-transform infrared (FTIR) spectroscopy was employed to investigate the interaction between nano-silica and caprolactam. The FTIR spectra of original nano-silica and silica/caprolactam compound were recorded in $\mathrm{KBr}$ pellets on a Nexus470 Fourier-transform IR spectrometer (Nicolet, United States of America). Blank scanning was performed before measurements to eliminate the influence of water vapor and $\mathrm{CO}_{2}$ in the air.

To assess nanoparticles dispersion in the composites matrix, ultrathin film was randomly cut from the inner part of composites and observed through a JEM-100CXII transmission electron microscope (TEM, Japan).

The wide angle X-ray diffraction (WAXD) analysis was conducted using a Bruker D8 Diffractometer (Germany). The X-ray beam was nickel-filtered $\mathrm{CuK}_{\alpha}$ radiation $(\lambda=0.15 \mathrm{~nm})$, and data were collected from 5 to $35^{\circ}(2 \theta)$ at a rate of $2 \%$ min.

Thermogravimetric analysis (TGA) measurements were performed using a TA-5200 thermogravimetric analyzer (United States of America) from 25 to $700^{\circ} \mathrm{C}$ with a heating rate of $20^{\circ} \mathrm{C} / \mathrm{min}$ under flowing of nitrogen.

The mechanical properties reported hereinafter are the average of five successful tests. All specimens were mechanically cut using a ZHY-W universal sample maker (CHENGDE Dahua Tester Co. Ltd., Hebei, China). Dumbbell specimens with waist dimensions of $25.0 \times 5.0 \times 2.0 \mathrm{~mm}$ were used for tensile tests in a SANS CMT-6104 (MTS, United States of America) universal tester. Notched impact strength were tested in XCJ-40 Charpy Tester (CHENGDE Materials Tester Co. Ltd., Hebei,
China), the dimensions of these samples were $40.0 \times 3.0 \times 2.0 \mathrm{~mm}$ (length $\times$ width $\times$ thickness). All the specimens were conditioned at $30^{\circ} \mathrm{C}$ and a relative humidity of $50 \%$ for $48 \mathrm{~h}$ prior to the measurements, which were then tested under the same conditions.

\section{Results and discussion}

\subsection{Interaction between nano-silica and caprolactam}

According to the structure (Figure 2), nano-silica has silanol groups on the surface, while caprolactam contains amide group. At the beginning of discussion, therefore, it should be studied whether there is interaction between nano-silica and caprolactam, since it is planned to use caprolactam as dispersion medium and stabilizer for separated nanoparticles against re-agglomeration.

Figure 3 gives the FTIR spectra of caprolactam and nano-silica/caprolactam. In the spectrum of neat caprolactam, the observed $\mathrm{N}-\mathrm{H}$ stretching band and the amide I mode known to be dominated by the $\mathrm{C}=\mathrm{O}$ stretching band are 3218 and $1660 \mathrm{~cm}^{-1}$ respectively. These two bands are very broad and strong due to the fact that caprolactam possesses typical self-associated hydrogen bonds formed

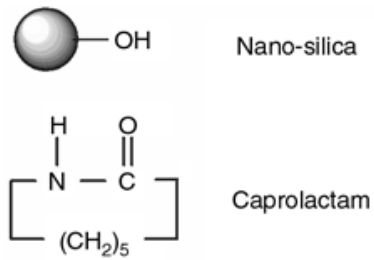

Figure 2. Schematic structure of nano-silica and caprolactam

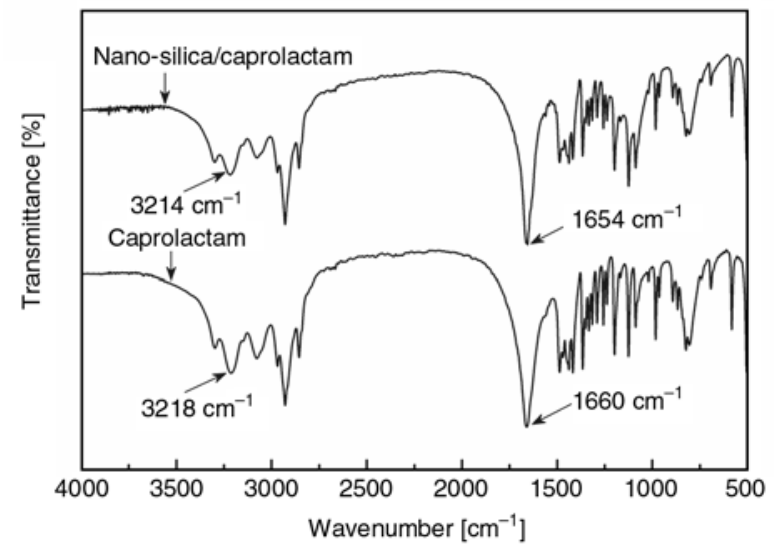

Figure 3. FTIR spectra of caprolactam and nanosilica/caprolactam compound (nano-silica content $=2.0 \mathrm{wt} \%$ ) 
between $\mathrm{C}=\mathrm{O}$ and $\mathrm{N}-\mathrm{H}$. For the spectrum of nanosilica/caprolactam, the absorption of $\mathrm{N}-\mathrm{H}$ (appears at $3214 \mathrm{~cm}^{-1}$ ) and $\mathrm{C}=\mathrm{O}$ (appears at $1654 \mathrm{~cm}^{-1}$ ) show obvious displacement to lower frequency compared to neat caprolactam. In consideration of the fact that there are plentiful silanol groups on the surface of nano-silica, the above results should indicate the existence of hydrogen bonds between caprolactam and nano-silica [17].

\subsection{Microstructural characterization}

Filler dispersion is of great importance in the physical behaviors of polymer composites. As shown by the TEM photos in Figure 4, the nanoparticles have homogeneous dispersion in matrix. The dispersion scale of the nano-silica in matrix ranges from 25 to $70 \mathrm{~nm}$, i.e. nanoparticles agglomerations were broken into smaller ones or even mono-dispersing particles since the original size of nano-silica is about $30 \pm 5 \mathrm{~nm}$ (see Experimental part). Clearly, this also manifests the feasibility of the strategy we proposed in the Introduction part to prepare silica/MC nylon6 in-situ nanocomposites with uniformly distributed nanoparticles.

Nylon6 is well known to crystallize into variously phase: the $\alpha$ stable crystal and among others the $\gamma$ one [18]. WAXD was used to gain insight into the influence of nano-silica on the crystal form of MC nylon6. As is shown in Figure 5, the peak at $2 \theta=$ $20.3^{\circ}$ belongs to the (200) face of the $\alpha 1 \mathrm{form}$, the peak at $2 \theta=21.6^{\circ}$ belongs to the (001), (200), and (201) faces of the $\gamma$ form, and the peak at $2 \theta=23.9^{\circ}$ belongs to the (002) and (202) faces of the $\alpha 2$ form. Therefore, it is evident that the $\alpha$ form is the dominant crystalline phase for both neat MC nylon6 and

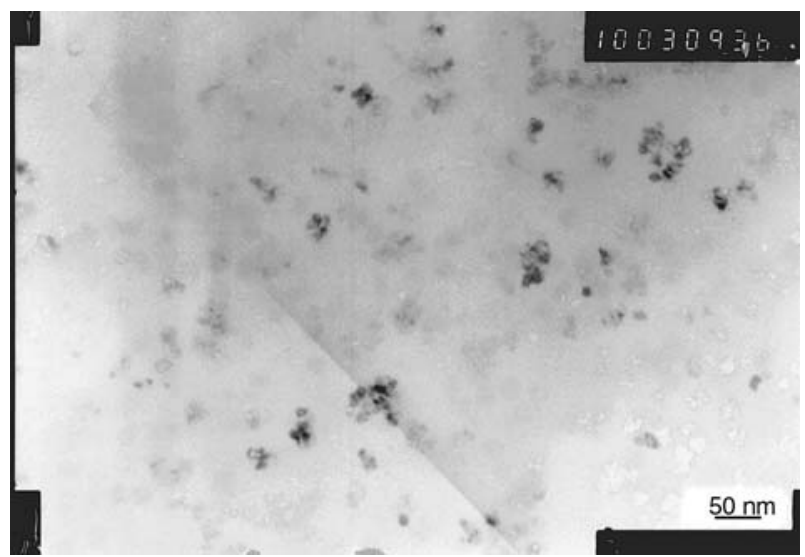

Figure 4. TEM image of a silica/MC nylon6 in-situ nanocomposites (nano-silica content $=2.0 \mathrm{wt} \%$ )

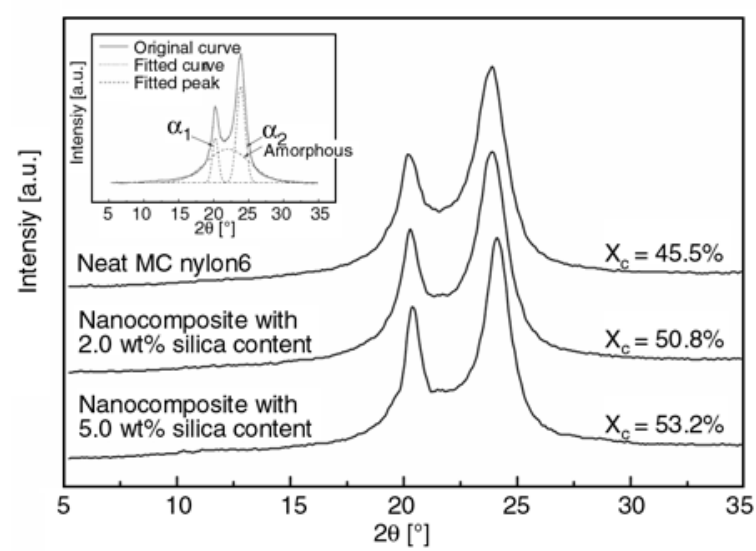

Figure 5. WAXD spectra of MC nylon6 and nanocomposites. The inset illustrates the WAXD curve treated by fitting and peak separation. $X_{c}$ denotes crystalliniy.

nanocomposites (Figure 5), which is consistent with the results reported by Reynaud et al. [8]. In order to further studied the crystallinity of neat MC nylon6 and nanocomposites, the WAXD curves were treated with fitting and peak separation as shown in the inset of Figure 5 to get $\alpha 1$ form, $\alpha 2$ form and amorphous. The crystalliniy $\left(X_{c}\right)$ was calculated according to Equation (1):

$$
X_{c}[\%]=\frac{\Sigma\left(A_{\alpha 1-\text { form }}+A_{\alpha 2-\text { form }}\right)}{\Sigma\left(A_{\alpha 1-\text { form }}+A_{\alpha 2-\text { form }}+A_{\text {amorphous }}\right)} \cdot 100
$$

where $A_{\alpha 1 \text {-form }}, A_{\alpha 2 \text {-form }}$ and $A_{\text {amorphous }}$ denote the area of various fitted peaks ( $\alpha 1$ form, $\alpha 2$ form and amorphous, respectively). As shown in Figure 5, the crystalliniy $\left(X_{c}\right)$ of nanocomposites is higher than that of neat MC nylon6, indicating the promotion of nano-silica on the crystallization of nylon6 matrix. Similar results were also reported in nylon6/clay nanocomposites, and such promotion behaviors were generally attributed to the nucleation effect of nano-fillers [19, 20].

\subsection{Thermal behavior of nanocomposties}

TGA weight loss obtained in a nitrogen atmosphere for the neat MC nylon6 and nanocomposites is shown in Figure 6. It can be seen that the neat MC nylon6 exhibits two-step weight loss mechanism, while the nanocomposites show delayed decomposition in first step compared to neat MC nylon6. The temperatures at which 10,50 , and $90 \%$ weight loss occur as representative of thermal stability are 
Table 1. TGA results of MC nylon6 and nanocomposites

\begin{tabular}{|l|c|c|c|c|c|}
\hline \multirow{2}{*}{} & \multirow{2}{*}{ MC nylon6 } & \multicolumn{4}{|c|}{ Nanocomposites with various silica content [wt\%] } \\
\cline { 3 - 6 } & & $\mathbf{1 . 0 \%}$ & $\mathbf{2 . 0 \%}$ & $\mathbf{3 . 0 \%}$ & $\mathbf{5 . 0 \%}$ \\
\hline$T_{10 \%}{ }^{*}\left[{ }^{\circ} \mathrm{C}\right]$ & 321.58 & 336.21 & 360.19 & 400.97 & 396.58 \\
\hline$T_{50 \%}{ }^{*}\left[{ }^{\circ} \mathrm{C}\right]$ & 440.89 & 437.76 & 435.36 & 444.16 & 444.16 \\
\hline$T_{90 \%}{ }^{\circ}\left[{ }^{\circ} \mathrm{C}\right]$ & 472.03 & 465.75 & 461.35 & 467.75 & 467.75 \\
\hline Residue $[\mathrm{wt} \%]$ & 0.02 & 1.12 & 2.03 & 2.92 & 5.10 \\
\hline
\end{tabular}

${ }^{*} T_{10 \%}, T_{50 \%}$ and $T_{90 \%}$ denote to the temperature at various weight loss of $10 \%, 50 \%$ and $90 \%$, which is calculated from the TGA thermograms

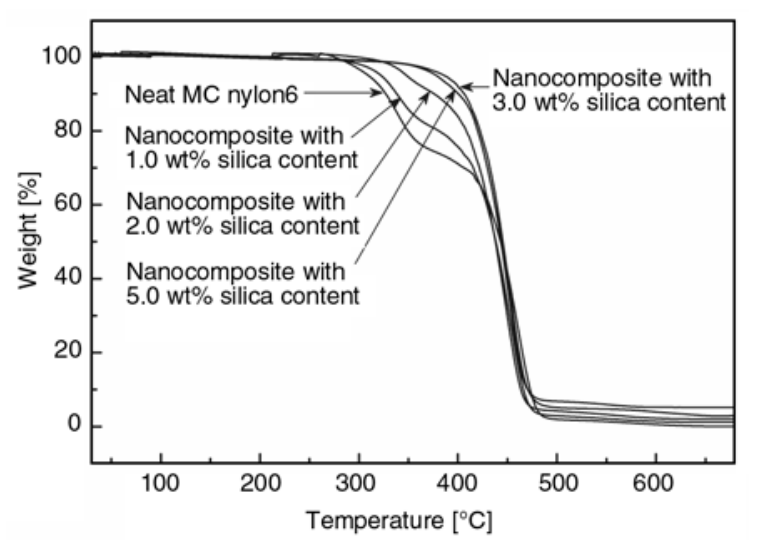

Figure 6. TGA thermograms of MC nylon6 and nanocomposites as a function of silica content

listed in Table 1. It is evident that the influence of nano-silica on thermal stability of MC nylon6 differs at different ranges of weight loss. At lower weight loss (10 and 50\%), the notable tendency of improved thermal stability of the nanocomposites with increasing nano-silica content can be seen from Table 1. At present, the detailed mechanism of thermal stabilization of the filled nylon6 systems is not clearly understood. According to Theng [21] and Velde [22], the interaction between the amide groups of nylon6 molecules and the ionic groups at the clay surfaces probably suppresses thermal degradation of the composite matrix. Such explanation may also be valid for the improved thermal stability of nanocomposites as mentioned above. However, it is interesting to find that thermal stability of nanocomposites is slightly inferior to neat MC nylon6 when higher weight loss (90\%) is considered. Such results indicate that the introduction of silica might change the decomposition mechanism of MC nylon6 under high temperature. Further work will be carried out on such high temperature decomposition mechanism of silica/MC nylon6 in-situ nanocomposites.

\subsection{Mechanical properties}

Figure 7 shows notched Charpy impact strength of nanocomposites as a function of nano-silica content. It is seen that the contribution of the uniformly distributed nanopartilces to the improvement of toughness under high speed deformation and to the reduction of notch sensitivity is notable, and the highest impact strength of composites (appears at $3.0 \mathrm{wt} \%$ of silica content) is about 1.17 times higher than that of neat MC nylon6. So far, the crack front bowing mechanism has been widely used for explaining the toughening effect in micro-sized particulate filled polymers [23]. Nevertheless, Chan et al. [24] inferred that in the case where the size of rigid particles is of the order of $50 \mathrm{~nm}$ or less, the applicability of the bowing mechanism is questionable, because such small size rigid particles may not be able to resist the propagation of the crack. From J-integral tests, they suggested that nanoparticles would trigger large-scale plastic deformation of the matrix, which consumes tremendous fracture energy. Since the above morphological investigation reveals that most of the dispersion scale of the

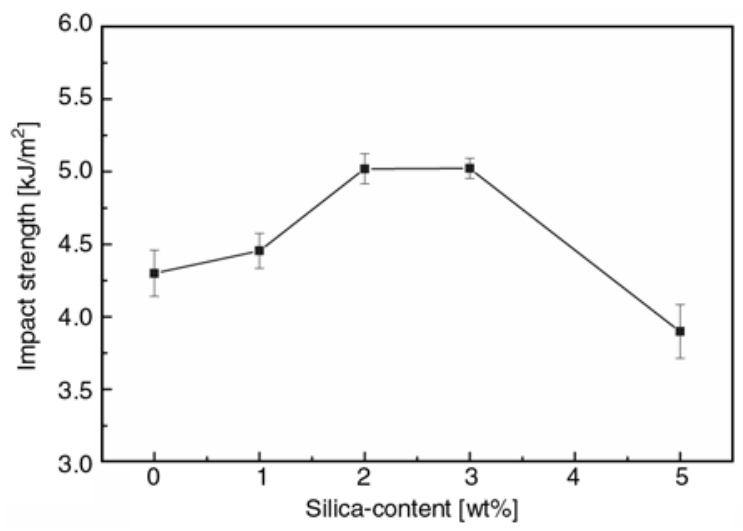

Figure 7. Notched Charpy impact strength of MC nylon6 and nanocomposites as a function of silica content 


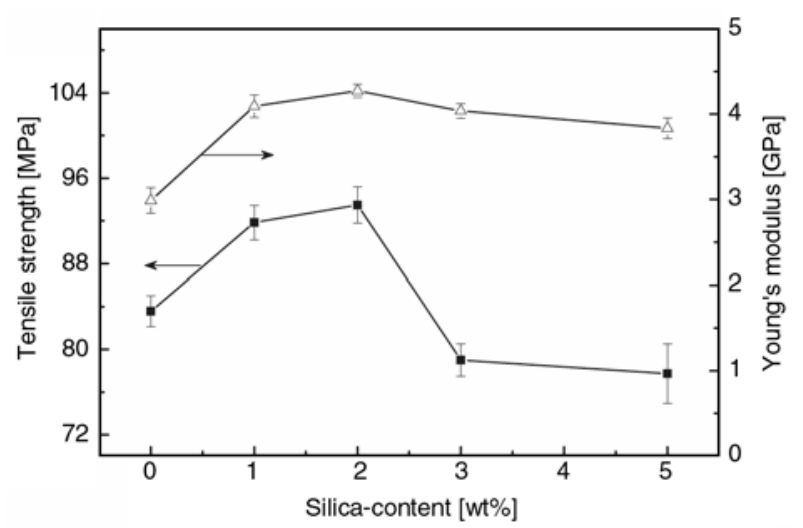

Figure 8. Tensile strength and Young's modulus of MC nylon6 and nanocomposites as a function of silica content

nanoparticles is less than $50 \mathrm{~nm}$ in the composites, it is believed that the mechanism proposed by Chan and co-workers should also be valid in the current study.

Tensile strength of nanocomposites as a function of nano-silica content is shown in Figure 8. In comparison with neat MC nylon6, the composites show prominent improvement of tensile strength under lower nano-silica content ( $2 \mathrm{wt} \%$ ), and the highest tensile strength of composites (appears at $2.0 \mathrm{wt} \%$ of silica content) is about 1.12 times higher than that of neat MC nylon6. According to the above FTIR results, there are hydrogen bonds between nano-silica and amide group. Such interaction should also account for the strengthening effect, as the stress was effectively transferred $[25,26]$. The filler content dependences of Young's modulus of the composites are also exhibited in Figure 8. In comparison with neat MC nylon6, all of the composites show higher Young's modulus. At a silica content of $2.0 \mathrm{wt} \%$, for example, the Young's modulus shows maximum value and is about 1.43 times higher than that of neat MC nylon6. Similarly, the interaction between nano-silica and caprolactam can effectively impart the rigidity of nanoparticles to MC nylon6.

\section{Conclusions}

On the basis of the above discussion, the following conclusions can be drawn.

1. Caprolactam can be used as dispersion medium and stabilizer for the separated nanoparticles against re-agglomeration owing to the existence of hydrogen bond between nano-silica and caprolactam.

2. TEM photos reveal a uniform distribution of nano-silica in matrix. This manifests that the nanoparticles aggregations were broken into smaller ones or even mono-dispersing particles according to the technology we proposed in this paper.

3. Within the sensitivity of our experimental instrument, no influence of nano-silica can be noticed on the crystal form in these MC nylon6 based nanocomposites. However, nano-silica shows obvious promotion effect on the crystallization of nylon6 matrix.

4. Thermal stability of nanocomposites prepared according to the technology we proposed in this paper is notably improved compared to neat MC nylon6. And nanocomposites also receive simultaneous improvement of toughness, strength and stiffness.

\section{Acknowledgements}

The authors are grateful to the support of National Natural Science Foundation of China (Grant: 59602007).

\section{References}

[1] Zhang M. Q., Rong M. Z., Friedrich K.: Processing and properties of nonlayered nanoparticle reinforced thermoplastic composites. in 'Handbook of organicinorganic hybrid materials and nanocomposites. Vol 2: Nanocomposites' (ed.: Nalwa H. S.) American Science Publishers, Boston, 113-150 (2003).

[2] Leuteritz A., Pospiech D., Kretzschmar B., Willeke M., Jehnichen D., Jentzsch U., Grundke K., Janke A.: Progress in polypropylene nanocomposite development. Advanced Engineering Materials, 5, 678-681 (2003).

DOI: $10.1002 /$ adem.200320139

[3] Rong M. Z., Zhang M. Q., Ruan W. H.: Surface modification of nanoscale fillers for improving properties of polymer nanocomposites: A review. Materials Science and Technology, 22, 787-796 (2006). DOI: $\underline{10.1179 / 174328406 X 101247}$

[4] Novak B. M.: Hybrid nanocomposite materialsbetween inorganic glasses and organic polymers. Advanced Materials, 5, 422-433 (1993). DOI: $10.1002 / \mathrm{adma} .19930050603$

[5] Cho J. W., Paul D. R.: Nylon 6 nanocomposites by melt compounding. Polymer, 42, 1083-1094 (2001). DOI: $10.1016 / \mathrm{S} 0032-3861(00) 00380-3$ 
[6] Dennis H. R., Hunter D. L., Chang D., Kim S., White J. L., Cho J. W., Paul D. R.: Effect of melt processing conditions on the extent of exfoliation in organoclaybased nanocomposites. Polymer, 42, 9513-9522 (2001).

DOI: 10.1016/S0032-3861(01)00473-6

[7] García M., van Vliet G., ten Cate M. G. J., Chavez F., Norder B., Kooi B., van Zyl W. E., Verweij H., Blank D. H. A.: Large-scale extrusion processing and characterization of hybrid nylon- $6 / \mathrm{SiO}_{2}$ nanocomposites. Polymers for Advanced Technologies, 15, 164-172 (2004).

DOI: $10.1002 /$ pat.458

[8] Reynaud E., Jouen T., Gauthier C., Vigier G., Varlet J.: Nanofillers in polymeric matrix: A study on silica reinforced PA6. Polymer, 42, 8759-8768 (2001). DOI: $10.1016 / \mathrm{S} 0032-3861(01) 00446-3$

[9] Paul D. R., Robeson L. M.: Polymer nanotechnology: Nanocomposites. Polymer, 49, 3187-3204 (2008). DOI: $10.1016 /$ j.polymer.2008.04.017

[10] Tadros T. F.: Industrial applications of dispersions. Advances in Colloid and Interface Science, 46, 1-47 (1993). DOI: 10.1016/0001-8686(93)80032-7

[11] James R. O.: Characterization of colloids in aqueous systems. in 'Ceramic powder science' (eds.: Messing G. L., Mazdiyansi K., Haber R. A.) American Ceramic Society, Westerville, 349-410 (1987).

[12] Rusu G., Rusu E.: Nylon 6/SiO 2 nanocomposites synthesized by in situ anionic polymerization. High Performance Polymers, 18, 355-375 (2006). DOI: $10.1177 / 0954008306063392$

[13] Joung G. R., Sang W. P., Kim H. S., Lee J. W.: Power ultrasound effects for in situ compatibilization of polymer-clay nanocomposites. Materials Science Engineering: C, 24, 285-288 (2003). DOI: $10.1016 /$ j.msec.2003.09.057

[14] Mougin N., Veith C. A., Cohen R. E., Gnanou Y.: Anionic polymerization of lactams in the presence of metal dialkoxyaluminum hydrides: Presentation of a new mechanism. Macromolecules, 25, 2004-2016 (1992).

DOI: $10.1021 / \mathrm{ma} 00033 \mathrm{a} 026$
[15] Rusu G., Ueda K., Rusu E., Rusu M.: Polyamides from lactams by centrifugal molding via anionic ringopening polymerization. Polymer, 42, 5669-5678 (2001)

DOI: $10.1016 / \mathrm{S} 0032-3861(01) 00059-3$

[16] Kircher K.: Chemical reaction in plastics processing. Hanser, Munich (1987).

[17] Xue Q.: Spectroscopy in the study of macromolecular structure (in Chinese). High Education Publishers, Beijing (1995).

[18] Lin L., Argon A. S.: Deformation resistance in oriented nylon 6. Macromolecules, 25, 4011-4024 (1992). DOI: $10.1021 / \mathrm{ma} 00041 \mathrm{a} 025$

[19] Jiang T., Wang Y-H., Yeh J-T., Fan Z-Q.: Study on solvent permeation resistance properties of nylon6/clay nanocomposite. European Polymer Journal, 41, 459466 (2005). DOI: $10.1016 /$ j.eurpolymj.2004.10.024

[20] Katoha Y., Okamoto M.: Crystallization controlled by layered silicates in nylon 6-clay nano-composite. Polymer, 50, 4718-4726 (2009). DOI: 10.1016/j.polymer.2009.07.019

[21] Theng B. K. G..: The chemistry of clay-organic reactions. Halsted Press, New York (1974).

[22] Velde B.: Introduction to clay minerals: Chemistry, origins, uses, and environmental significance. Chapman and Hall, London (1992).

[23] Lange F. F.: The interaction of a crack front with a second-phase dispersion. Philosophical Magazine, 22, 983-992 (1970). DOI: $10.1080 / 14786437008221068$

[24] Chan C-M., Wu J-S., Li J-X., Cheung Y-K.: Polypropylene/calcium carbonate nanocomposites. Polymer, 43, 2981-2992 (2002). DOI: $10.1016 / \mathrm{S} 0032-3861(02) 00120-9$

[25] Cai L. F., Huang X. B., Rong M. Z., Ruan W. H., Zhang M. Q.: Effect of grafted polymeric foaming agent on the structure and properties of nano-silica/ polypropylene composites. Polymer, 47, 7043-7050 (2006). DOI: 10.1016/j.polymer.2006.08.016

[26] Cai L. F., Mai Y. L., Rong M. Z., Ruan W. H., Zhang M. Q.: Interfacial effects in nano-silica/polypropylene composites fabricated by in-situ chemical blowing. Express Polymer Letters, 1, 2-7 (2007). DOI: $10.3144 /$ expresspolymlett.2007.2 